\title{
Fast Nonlinear Deterministic Calibration of Pipelined A/D Converters
}

\author{
Takashi Oshima ${ }^{\dagger}$ Tomomi Takahashi ${ }^{\dagger}$ Taizo Yamawaki ${ }^{\dagger}$ Cheonguyen Tsang ${ }^{\ddagger}$ Dusan Stepanovic ${ }^{\ddagger}$ Borivoje Nikolic ${ }^{\ddagger}$ \\ $\dagger$ Hitachi Ltd., Central Research Laboratory, 1-280 Higashi-koigakubo, Kokubunji-shi, Tokyo 185-8601, Japan \\ † Berkeley Wireless Research Center of University of California at Berkeley \\ 2108 Allston Way, Suite 200 Berkeley, CA 94704-1302, USA
}

\begin{abstract}
The high-speed and high-resolution ADC is a key enabler for many future wireless communications systems. The digital background calibration technique can be used to reduce the total power consumption by enhancing the linearity without using high-gain amplifiers. One of the main practical constraints in the wireless applications is a short time available for calibration. This paper proposes a novel fast calibration method of pipelined ADCs, suitable for wireless communications applications, where a sufficiently high resolution can be achieved without requiring any calibration period.
\end{abstract}

Keyword Pipelined ADC, digital calibration, convergence time, MDAC, redundancy.

\section{Introduction}

Many of the advances in future wideband communications systems require the development of high-speed high-resolution A/D converters. However, there is a fundamental tradeoff between the dynamic range, bandwidth and power dissipation in ADC's. Presently, pipelined A/D architectures achieve the lowest power for 10-12-bit resolutions and several hundreds of $\mathrm{MHz}$ of bandwidth. By using the digital calibration, it is possible to relax the tradeoff between the resolution and speed, allowing for a significant reduction in power. A method proposed in [1] achieves fast convergence of calibration by using a reference converter in addition to the main $\mathrm{A} / \mathrm{D}$ converter. Its inherently deterministic nature enables superior convergence to the other methods relying on the random statistics of pseudo-noise sequences [2-4]. The overall structure of $\mathrm{A} / \mathrm{D}$ calibration from [1] is shown in Fig. 1. It consists of main pipeline ADC with $1.5 \mathrm{~b} / \mathrm{stage}$ MDAC's, reference ADC, digital-background-calibration block, digital error correction (DEC) and front-end $\mathrm{S} / \mathrm{H}$ circuit. The effective resolution of both ADCs should be larger than the target resolution of the overall $\mathrm{A} / \mathrm{D}$ conversion by 1 or 2 bits. The op-amps used in MDAC of the main ADC need to have large GBW but are not necessarily required of a large open-loop gain, allowing the use of intrinsically faster, lower-gain, deeply-scaled CMOS. The small op-amp open-loop gain makes the output of the main A/D converter inaccurate, which must be corrected through digital background calibration. Consider the case where the open-loop gain of the op-amp for each MDAC is limited to only $30 \mathrm{~dB}$ for lower power consumption. The gain of MDAC decreases from the ideal value 2 to $G(\sim 1.88)$ but it doesn't cause any inaccuracy if digital error correction (DEC) is performed appropriately as shown in (1),

$V_{\text {out }}{ }^{D}=\sum_{i=1}^{N+1} w_{i} \cdot D_{i}=D_{1}+\frac{1}{G} \cdot D_{2}+\left(\frac{1}{G}\right)^{2} \cdot D_{3}+\ldots$ (1),

where $\mathrm{V}_{\text {out }}{ }^{\mathrm{D}}$ is the output of $\mathrm{A} / \mathrm{D}$ conversion and $\mathrm{D}_{\mathrm{i}}$ is a roughly-quantized output of each MDAC stage. Because of the process variations, the actual gain $G$ cannot be predicted accurately in advance, and hence it has to be determined. The approach proposed in [1] adopts the LMS algorithm in order to reach the most appropriate value of DEC coefficient $w_{i}$ with help by the reference $A / D$ converter. Although the reference A/D converter must have large effective resolution, it can operate in $\mathrm{K}$ times slower than the main A/D converter. Hence the power consumption of the reference $\mathrm{A} / \mathrm{D}$ converter can be decreased to a very low level. It provides the main A/D converter with the accurate conversion results $d$ of the analog input voltage $\mathrm{V}_{\text {in }}{ }^{\mathrm{A}}$ once in $\mathrm{K}$ samples. The output of the overall $\mathrm{A} / \mathrm{D}$ converter $\mathrm{V}_{\text {out }}{ }^{\mathrm{D}}$ is calculated by the digital error correction DEC using the current values of coefficient $\mathrm{w}_{\mathrm{i}}, \mathrm{w}_{\mathrm{i}}{ }^{(\mathrm{n})}$ and the output of the main $\mathrm{A} / \mathrm{D}$ converter, $\mathrm{D}_{\mathrm{i}}$ as follows,

$V_{\text {out }}{ }^{D}=\sum_{i=1}^{N+1} w_{i}^{(n)} \cdot D_{i}$

The current conversion error e between $\mathrm{V}_{\text {out }}{ }^{\mathrm{D}}$ and the output $\mathrm{d}$ of the reference $\mathrm{A} / \mathrm{D}$ converter which is supposed to be the correct conversion result of $\mathrm{V}_{\text {in }}{ }^{\mathrm{A}}$ is calculated by a subtraction once in $\mathrm{K}$ samples.

$e=d-V_{\text {out }}{ }^{D}$

Finally, the values of $\mathrm{w}_{\mathrm{i}}^{(\mathrm{n})}$ are updated to $\mathrm{w}_{\mathrm{i}}{ }^{(\mathrm{n}+1)}$ following (4) which are going to be used for the estimation of $V_{\text {out }}{ }^{D}$ of the next $\mathrm{K}$ samples. 


$$
w_{i}^{(n+1)}=w_{i}^{(n)}+\mu \times D_{i} \times e
$$

The negative feedback nature of the algorithm makes all $\mathrm{w}_{\mathrm{i}}(\mathrm{i}=1 \sim \mathrm{N}+1)$ converge to the most appropriate values automatically. The important feature of LMS method is that each update is proportional not only to the error e but also to data $D_{i}$ as shown in (4), which greatly enhances convergence ability. For correct operation, this algorithm needs to be exercised with sufficiently scattered values of $\mathrm{D}_{\mathrm{i}}$, which requires the full scale analog input $\mathrm{V}_{\text {in }}{ }^{\mathrm{A}}$. However, in many wireless systems, the signal is often weak or even absent most of the time, making this calibration approach impractical.

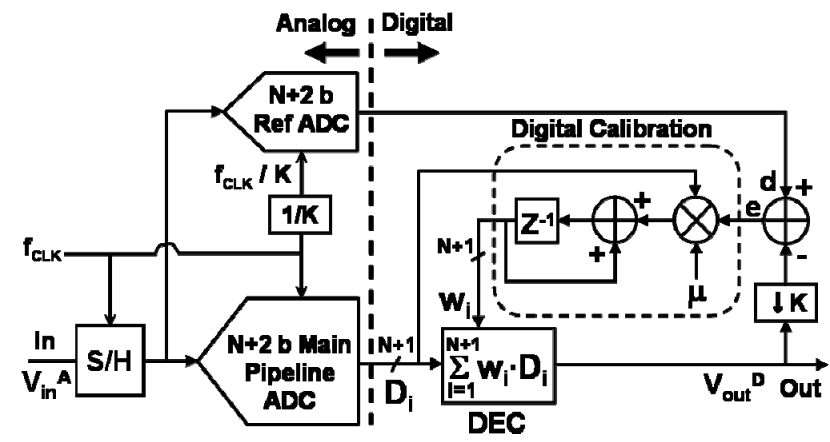

Fig. 1 LMS-calibration A/D converter with reference converter

\section{Proposed Fast Calibration Method}

The proposed calibration is based on a modification of the method described above. It exploits a dithered MDAC operation as shown in Fig. 2. It is known that digital fractional values can be added right after sub-ADC(SADC) without affecting the final $\mathrm{A} / \mathrm{D}$ conversion results, as long as the output of MDAC is within the full-scale range. The proposed method exploits this inherent redundancy by adding one of the three values $-\alpha, \quad 0,+\alpha$ to the node periodically (i.e. deterministically) depending on which segment $\left(\mathrm{V}_{\text {in }}<\right.$ $\left.-\mathrm{V}_{\text {ref }} / 4,-\mathrm{V}_{\text {ref }} / 4<\mathrm{V}_{\text {in }}<\mathrm{V}_{\text {ref }} / 4, \mathrm{~V}_{\text {ref }} / 4<\mathrm{V}_{\text {in }}\right)$ the input voltage $\mathrm{V}_{\text {in }}$ belongs to. This ternary or 1.5 -b deterministic dithering helps MDAC output $\mathrm{D}_{\mathrm{i}}{ }^{\text {'s }} \mathrm{s}$ of the main $\mathrm{A} / \mathrm{D}$ converter experience variety of values even in the case of zero input signal and thus ensures the excellent convergence of calibration without affecting the final conversion accuracy. The advantage described above is evident in Fig. 3(b). The proposed dithering enables the convergence of calibration even before the arrival of the preamble of packet (Pr) which is usually used for various calibrations or automatic gain control of the entire wireless receiver. On the other hand, the calibration without dithering cannot converge even within the preamble period since it cannot work while input signal is absent and hence begins to work only after the preamble begins to be received as shown in Fig. 3(a). Fig. 4 shows MDAC circuit which can implement the concept of Fig. 2. The third capacitance $\mathrm{C}_{3}$ is required in addition to $\mathrm{C}_{1}$ and $\mathrm{C}_{2}$ used in the conventional MDAC to implement the dithering. The capacitance $\mathrm{C}_{3}$ is chosen to be $\alpha$ times as large as $C_{1}$ and $C_{2}$ and hence $C_{3}$ is smaller than these capacitances.

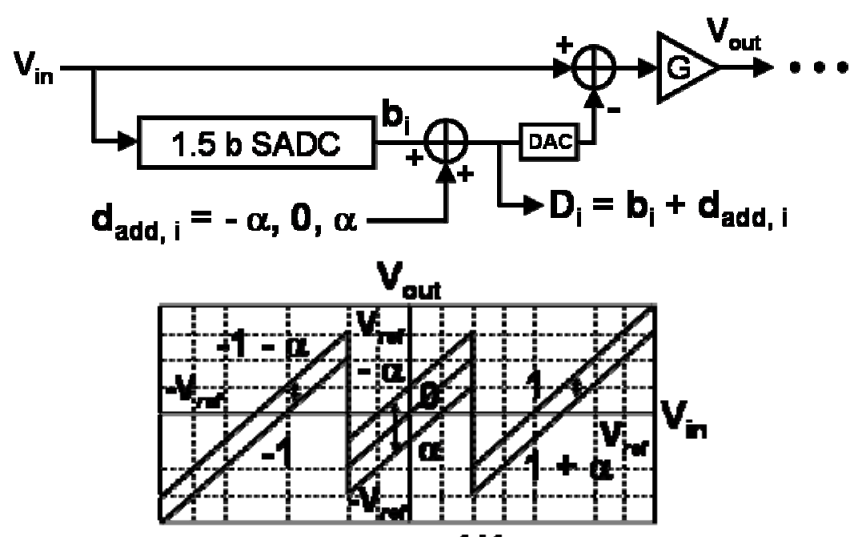

e.g. $\alpha=1 / 4$

Fig. 2 Proposed 1.5-b dithering of MDAC

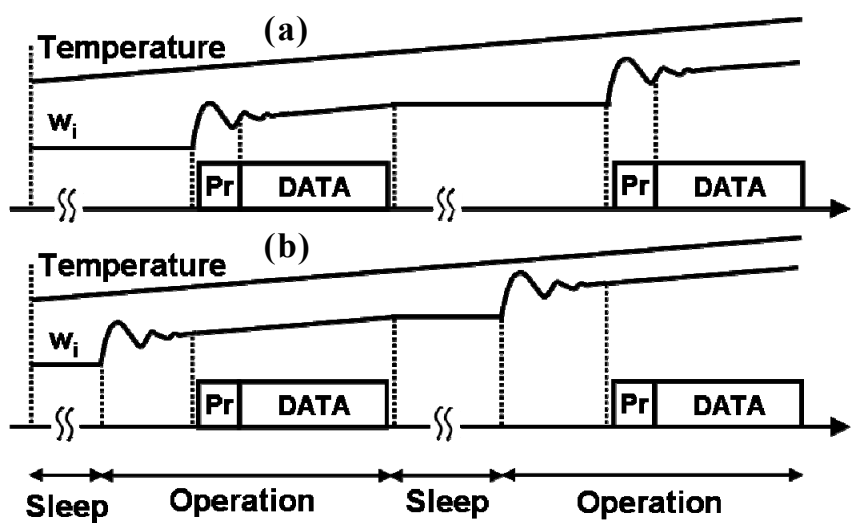

Fig. 3 Convergence behavior of calibration (a) without and (b) with proposed dithering

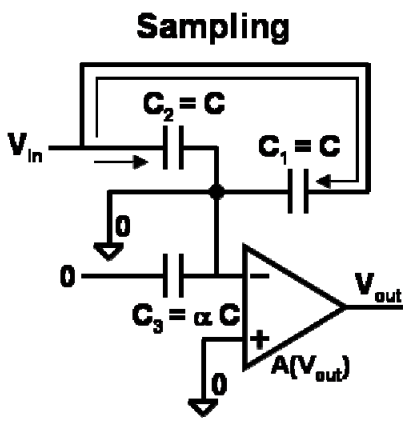

Amplifying (e.g. $\left.D_{\mathrm{i}}=1-\alpha\right)$

Fig. 4 Proposed MDAC circuit with internal dithering 


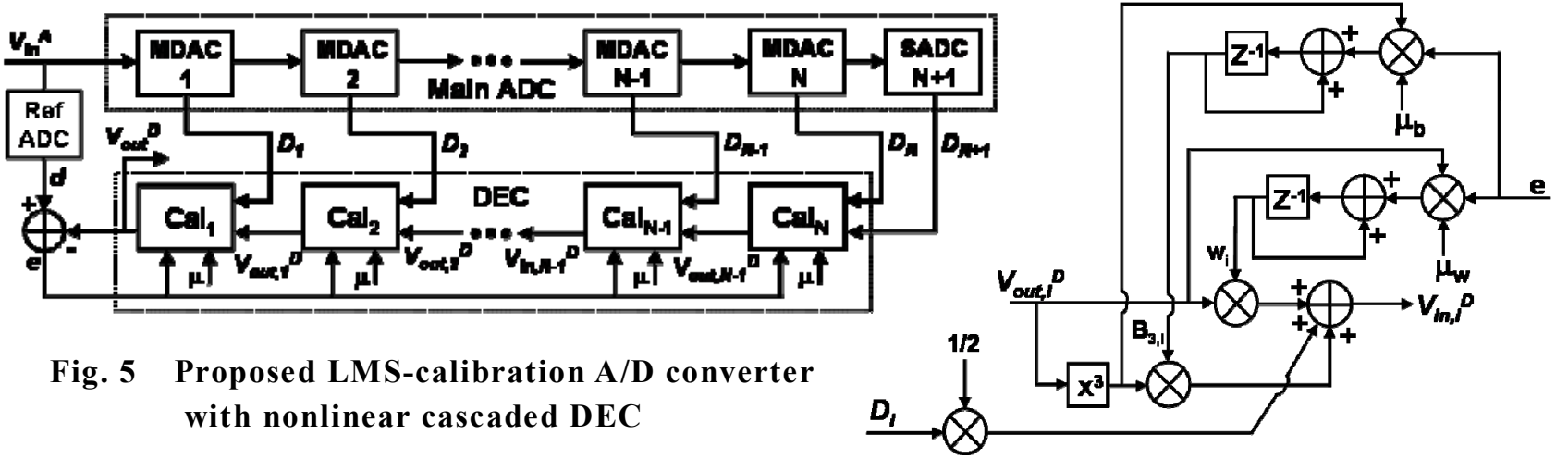

Fig. 6 Structure of each calibration stage

$$
\begin{aligned}
& V_{\text {out }}=2 \cdot \frac{1}{1+\left(2+\alpha+\frac{C_{X}}{C_{1}}\right) \cdot \frac{1}{A\left(V_{\text {out }}\right)} \cdot\left\{V_{\text {in }}-D_{i} \cdot \frac{1}{2} V_{\text {ref }}+\left(\frac{2+\alpha}{2}+\frac{C_{X}}{2 C_{1}}\right) \cdot V_{\text {os }}\right\}} \quad D_{i}=b_{i}+d_{\text {add }, i} \\
& A\left(V_{\text {out }}\right)=A_{0}+A_{2} \cdot V_{\text {out }}{ }^{2}+A_{4} \cdot V_{\text {out }}{ }^{4}+A_{6} \cdot V_{\text {out }}{ }^{6}+\cdots \quad \text { (6) } \\
& V_{\text {in }} \cong w \cdot V_{\text {out }}+B_{3} \cdot V_{\text {out }}{ }^{3}+B_{5} \cdot V_{\text {out }}{ }^{5}+D_{i} \cdot \frac{1}{2} V_{\text {ref }}-\left(\frac{2+\alpha}{2}+\frac{C_{X}}{2 C_{1}}\right) \cdot V_{\text {os }} \quad \text { (7) } \quad B_{3}=-\frac{A_{2}}{2 A_{0}^{2}}\left(2+\alpha+\frac{C_{X}}{C_{1}}\right) \\
& w=\frac{1}{2}\left\{1+\frac{1}{A_{0}}\left(2+\alpha+\frac{C_{X}}{C_{1}}\right)\right\}
\end{aligned}
$$

\section{Proposed Cascaded Nonlinear Calibration}

Proposed ADC with cascaded nonlinear calibration shown in Fig. 5 and 6 is based on the structure of Fig. 1 but was modified to calibrate not only the small-signal open-loop gain $\mathrm{A}_{0}$ but also the gain dependency on $\mathrm{V}_{\text {out }}, \mathrm{A}\left(\mathrm{V}_{\text {out }}\right)$ or the nonlinearity of op-amps in the context of LMS calibration described so far. When we include the parasitic capacitance $C_{X}$ and the offset voltage $V_{\text {os }}$ at the input of op-amp, the output $\mathrm{V}_{\text {out }}$ of MDAC is described as (5). The open-loop gain of op-amp can be described as a polynomial of op-amp output voltage $\mathrm{V}_{\text {out }}$ as (6). From (5) and (6) the input voltage $V_{\text {in }}$ of MDAC can be described as the polynomial of output voltage $V_{\text {out }}$, linear term of $D_{i}$ and DC offset as (7). As shown in (8), the coefficient w of $\mathrm{V}_{\text {out }}$ linear term, which corresponds to $\mathrm{w}_{\mathrm{i}}$ in Fig. 1, deviates from ideal value of $1 / 2$ due to the finite small-signal gain $\mathrm{A}_{0}$ of op-amp. The $3^{\text {rd }}$-order and the $5^{\text {th }}$-order terms of $\mathrm{V}_{\text {out }}$ are determined by the nonlinearity of the op-amp as (9) and (10). It should also be noted that the coefficient of $D_{i}$ is not affected by the open-loop gain of op-amp. Noticing the input voltage $\mathrm{V}_{\text {in }}{ }^{\mathrm{A}}$ of the main pipeline $\mathrm{A} / \mathrm{D}$ converter can be reconstructed in digital form $V_{\text {out }}{ }^{D}$ by repeating the calculation of (7) from the last to the $1^{\text {st }}$ stage in reverse using each MDAC output $D_{i}$, the digital error correction DEC can be realized by simply cascading the hardware implementation of (7). Keeping in mind the above, the overall $\mathrm{A} / \mathrm{D}$-conversion architecture is implemented as in Fig. 5, where each processing stage of DEC has the structure of Fig. 6, which is a straightforward implementation of (7) accommodating up-to $3^{\text {rd }}$-order nonlinear term in addition to conventional linear term. Since we cannot predict exact op-amp gain $\mathrm{A}\left(\mathrm{V}_{\text {out }}\right)$ in advance, the coefficients $\mathrm{w}$ and $\mathrm{B}_{3}$ are estimated by LMS algorithm, where each update of $w$ or $\mathrm{B}_{3}$ is proportional not only to the conversion error e but to $\mathrm{V}_{\text {out }}{ }^{\mathrm{D}}$ or $\mathrm{V}_{\text {out }}{ }^{\mathrm{D} 3}$ respectively as shown in Fig. 6.

\section{Simulation Results}

The proposed deterministic dithering and cascaded nonlinear calibration were verified by macro-based simulation for 12-bit A/D converter (i.e. $\mathrm{N}=10$ in Fig. 5). The equation (5) was exactly calculated in this simulation based on the model of $\mathrm{A}\left(\mathrm{V}_{\text {out }}\right)$ so that it give basically the same results as the circuit-level simulation as long as we assume the response time of MDAC is quick enough to ensure the relationship of (5). Fig. 7 compares the results of the calibration speed obtained with and without the proposed deterministic dithering in the case of low-gain op-amp with $\mathrm{A}\left(\mathrm{V}_{\text {out }}\right)=20$, where $\alpha$ was set to be $1 / 4$ and the dither $d_{\text {add }}$ was set to change periodically among $-\alpha, 0$, $+\alpha$. The case without dithering requires 5000 samples (denoted by arrow) for the convergence of the linear coefficient $\mathrm{w}_{1}$ of $1^{\text {st }}$ DEC stage and the conversion error $\mathrm{e}$ 
counting from the start of the packet because the calibration begins to work only after the signal begins to be received. This corresponds to the convergence time of $500 \mu$ s assuming the sampling rate of the reference converter to be $10 \mathrm{MS} / \mathrm{s}$, which is much longer than preamble periods of many wireless systems and hence not acceptable. On the other hand, since the calibration with proposed dithering can converge even in the absence of the signal, it already reaches the convergence by the time the packet begins to be received and therefore it doesn't require any fraction of the preamble for convergence. It should also be noted that $\mathrm{w}_{1}$ converges exactly to the value expected from (8). Fig. 8 shows the convergence behavior and the output spectrum in the case of nonlinear op-amp model $\mathrm{A}\left(\mathrm{V}_{\text {out }}\right)=20-4 \mathrm{~V}_{\text {out }}{ }^{2}$ with only-linear or up-to- $3^{\text {rd }}$-order nonlinear calibration in Fig. 6. SNDR was improved by $25 \mathrm{~dB}$ with linear calibration and by $8 \mathrm{~dB}$ further with $3^{\text {rd }}$-order nonlinear calibration. These simulation results confirm that both the proposed deterministic dithering for accelerating the convergence of calibration and the cascaded nonlinear digital calibration are working successfully.

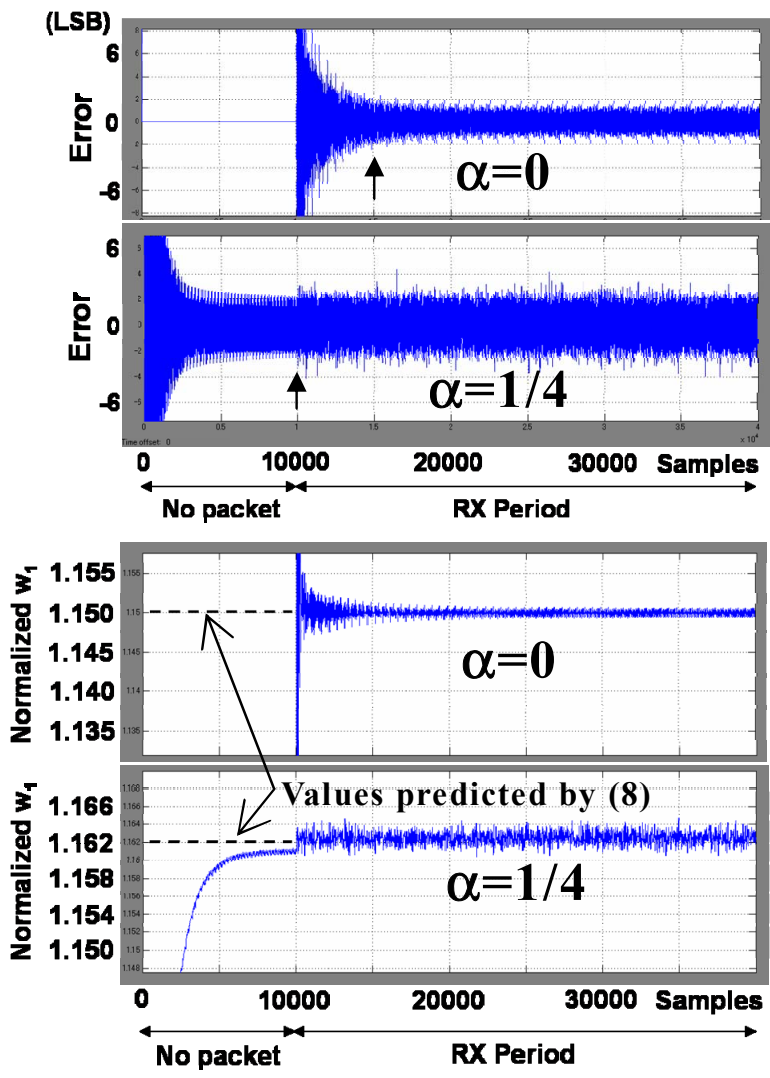

Fig. 7 Convergence behavior of A/D-conversion error and coefficient $w_{1}$ with and without dithering

\section{Reference}

[1] Y. Chiu et al., "Least mean square adaptive digital background calibration of pipelined analog-to-digital converters," IEEE Transactions on Circuits and Systems I Vol. 51, pp. 38-46(2004).

[2] J. Li et al., "Background calibration techniques for multistage pipelined ADCs with digital redundancy," IEEE Trans. on circuits and systems II, Vol. 50, pp. 531-538(2003).

[3] J. Keane et al., "Digital background calibration for memory effects in pipelined analog-to-digital converters," IEEE Trans. on circuits and systems I, Vol. 53, pp. 511-525(2006)

[4] B. Hernes et al., "A $92.5 \mathrm{~mW} 205 \mathrm{MS} / \mathrm{s} 10 \mathrm{~b}$ pipeline IF ADC implemented in $1.2 \mathrm{~V} / 3.3 \mathrm{~V} \quad 0.13 \mathrm{um}$ CMOS," ISSCC2007 Session 25.6(2007).

(L

(a)
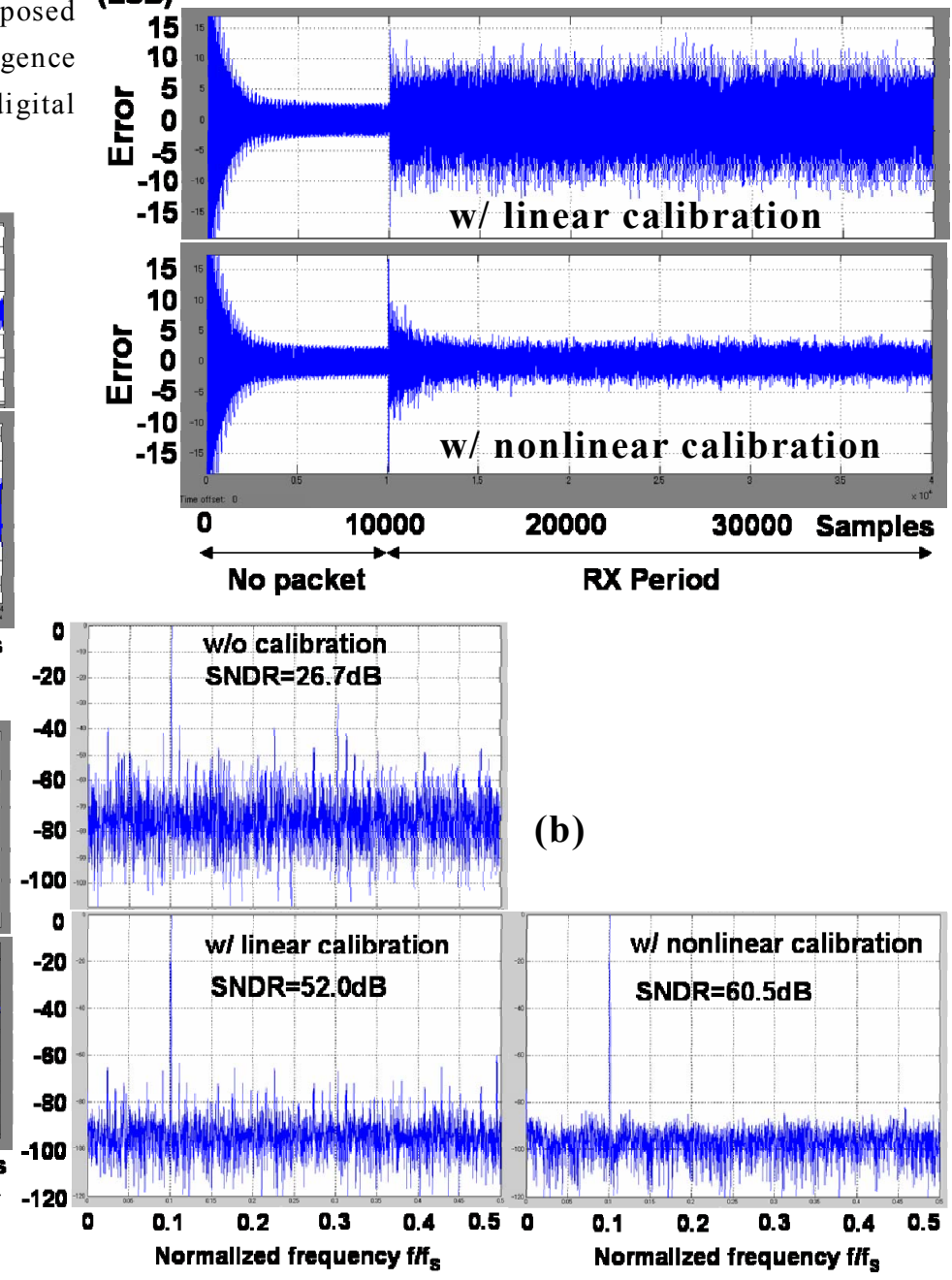

Fig. 8 (a) Convergence behavior and (b) Output spectrum with linear or nonlinear calibration 